\title{
Code Index Modulation: A High Data Rate and Energy Efficient Communication System
}

\author{
Georges Kaddoum, Mohammed F. A. Ahmed, and Yogesh Nijsure
}

\begin{abstract}
A new scheme, called code index modulation-spread spectrum (CIM-SS), is presented in this paper. This scheme is based on direct sequence-spread spectrum (DS-SS) modulation and can achieve higher data rate than the conventional DS-SS with lower energy consumption. At the transmitter, the bit stream is divided into blocks of two bits each. For each block, only the first bit is spread by one of two spreading codes while the second bit selects the code to be used. The receiver estimates both the code and the transmitted bit. The performance of the CIM-SS is analyzed and compared to the conventional DS-SS systems.
\end{abstract}

Index Terms-CIM-SS, High data rate, Performance analysis.

\section{INTRODUCTION}

One fundamental goal of wireless communications research is to attain the best utilization of the radio spectrum [1]. This is due to the growth in demand for data rates with the increased of the number of networked devices and the quality and types of wireless services. Additionally, wireless technologies are being utilized in variety of new scenarios which are characterized by low energy consumption such as wireless body area networks (WBANs) [2] and wireless sensor networks (WSNs) [3]. Consequently, innovative schemes should be developed for the future wireless technologies that achieve both high data rate and energy efficiency. For example, continuous phase modulation (CPM) is a constant envelope digital modulation scheme with good bandwidth and energy-efficiency [4].

One key technology that significantly increases the throughput of wireless channel is the multi-input multi-output (MIMO) [5]. In this scheme, the transmitter and receiver are mounted with multiple antennas to capture the spatial diversity of rich scattering environment. Spatial modulation (SM) is proposed as a low complexity variation to the MIMO scheme where the information bits are mapped to the constellation symbols and the transmit antenna index and only one antenna is needed for the transmission in each time slot [6]. This setting achieves high data rate with lower complexity as compared to the conventional MIMO and avoids inter-channel interference (ICI) and antenna synchronisation.

Orthogonal frequency-division multiplexing (OFDM) is adopted to combat frequency selective fading due to multipath propagation. Sub-carrier-index modulation OFDM (SIMOFDM) system is proposed in [7] where only a subset of sub-carriers is used to modulate the information bits. The index of the active sub-carriers is considered as an additional dimension and maps the information in an on-off keying

G. Kaddoum, M. F. A. Ahmed, and Y. Nijsure are with University of Québec, ETS engineering school, LACIME Laboratory, Montreal, Canada (email: georges.kaddoum@etsmtl.ca). fashion. An enhanced sub-carrier index modulation OFDM (ESIM-OFDM) scheme is shown in [8]. Each index modulated bit is mapped into two sub-carriers, with only one of them is active depending on the bit value.

In DS-CDMA (direct-sequence code division multiple access) scheme, orthogonal channels are created using orthogonal codes to support multiple users at the same time and frequency. For better performance, code-aided direct sequence spread spectrum (DS-SS) [9] and practical low-rate turboHadamard coding techniques [10] are proposed for narrowband interference suppression. The latter system shows better bit error rate (BER) performance than the code-aided DS-SS [9].

In this paper, we propose a new domain, namely the spreading code domain, aiming at increasing the data rate without adding extra computations complexity to the system. The proposed system, which is called code index modulationspread spectrum (CIM-SS), can be integrated with the MIMO or SM systems to achieve higher throughput. It uses spreading codes to map the data in conjunction with the constellation symbols. Specifically, for each pair of bits, one bit is spread by one spreading code, out of two, selected based on the value of the second bit. At the receiver, the spreading code is first detected which allow to recover the mapped bit then the transmitted bit is demodulated. With this elegant scheme, only half of the bits are physically transmitted over the channel and the other half are mapped into the spreading codes. Therefore, CIM-SS increases the throughput and decreases the energy consumption while keeping the system easy to implement. Moreover, the advantages of the spread spectrum modulation are maintained in the proposed CIM-SS scheme. Due to space limitation, and since the performance analysis is not straightforward, we develop an approximate BER expression over additive white Gaussian noise (AWGN) channel to validate the system design. Then, we provide performance comparison with direct sequence-spread spectrum (DS-SS) systems over AWGN and Rayleigh fading channels by simulations.

\section{SySTEM MODEL}

The system model for the CIM-SS is shown in Fig. 1. The transmitter employs quadrature phase-shift keying (QPSK) modulation and DS-SS. The information bits, which take the values $\{-1,+1\}$, are divided between in-phase and quadrature-phase branches and arranged into blocks of two bits each. Since the in-phase and quadrature-phase components are similar, we consider the in-phase component in the following analysis and the expressions for the quadraturephase component will be the same. For the $i$ th in-phase block

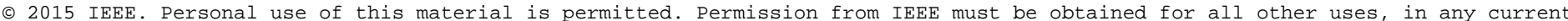

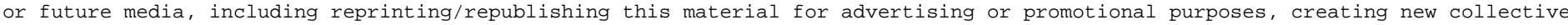
works, for resale or redistribution to servers or lists, or reuse of any copyrighted component of this work in other works. 
$S_{I, i}=\left(a_{1, i}, a_{2, i}\right)$, the first bit $a_{1, i}$ is multiplied by a spreading code selected by the second bit $a_{2, i}$. Since $a_{2, i}$ have two values (i.e. $a_{2, i} \in\{-1,+1\}$ ), two different orthogonal codes can be selected such as $w_{1}(t)$ if $a_{2, i}=1$ or $w_{2}(t)$ if $a_{2, i}=-1$. Walsh codes are chosen here due to their perfect orthogonality properties. Hence, the transmitted in-phase CIM-SS signal $e_{I}(t)$ is expressed as

$$
e_{I}(t)=\sum_{i=1}^{N} \sum_{k=1}^{L} a_{1, i} w_{a_{2, i}, k} p\left(t-(i L+k) T_{c}\right) \cos \left(2 \pi f_{0} t\right),
$$

where $N$ is the number of transmitted blocks, $w_{a_{2, i}, k} \in$ $\left\{w_{1, k}, w_{2, k}\right\}$ is the Walsh code chip sequence with chip period $T_{c}$ and length $L$ chips, $f_{0}$ is the carrier frequency, and $p(t)$ is the pulse shaping filter which is a rectangular pulse of unit amplitude on $\left[0, T_{c}\right]$. Note that $w_{a_{2, i}, k}$ is selected by $a_{2, i}$ to spread $a_{1, i}$, thus we denote $a_{1, i}$ as the transmitted bit and $a_{2, i}$ as the mapped bit. Since each transmitted bit $a_{1, i}$ contains a mapped bit $a_{2, i}$ in the spreading code selection, the proposed CIM-SS doubles the data rate for the same bandwidth and reduces $50 \%$ of the transmitted energy as compared to the conventional SS-QPSK system.

The receiver has two branches for the in-phase signal, one for each spreading code, as shown in Fig. 1 (b). The received in-phase signal is given by

$$
r_{I}(t)=e_{I}(t)+n(t),
$$

where $n(t)$ is an AWGN with zero mean and variance equal to $N_{0} / 2$. After baseband signal recovery and sampling, a correlator is used to detect the spreading code. The received sampled signal is multiplied at each branch by the corresponding Walsh code and summed over the bit duration $L T_{c}$. Therefore, the output at the first branch $I_{1, i}$ can be expressed as

$$
I_{1, i}=\left\{\begin{array}{c}
a_{1, i} E_{b}+n_{1}, w_{1} \text { transmitted ( i.e } a_{2, i}=1 \text { ), } \\
n_{1}, w_{2} \text { transmitted ( i.e } a_{2, i}=-1 \text { ), }
\end{array}\right.
$$

where $E_{b}=\sum_{k=1}^{L} w_{1, k}^{2}$ is the transmitted bit energy and $n_{1}=$ $\sum_{k=1}^{L} n_{k} w_{1, k}$ is the AGWN multiplied by the first Walsh code. In similar way, the output at the second branch $I_{2, i}$ can be found as

$$
I_{2, i}=\left\{\begin{array}{cl}
n_{2}, & \left., w_{1} \text { transmitted ( i.e } a_{2, i}=1\right), \\
a_{1, i} E_{b}+n_{2}, & \left., w_{2} \text { transmitted ( i.e } a_{2, i}=-1\right),
\end{array}\right.
$$

where $n_{2}=\sum_{k=1}^{L} n_{k} w_{2, k}$ is the corresponding noise term.

The absolute values of the correlator's output are compared in order to detect transmitted Walsh code based on the decision variable $D_{i}=\left|I_{1, i}\right|-\left|I_{2, i}\right|$ and hence the mapped bit $a_{2, i}$ is found. The corresponding branch is then selected by the comparator and used to recover the transmitted bit $a_{1, i}$ through the sign of its correlator output. Thus, the two bits can be recovered as follows

$$
\begin{aligned}
& D_{i}>0, \quad a_{2, i}=1, \text { and } a_{1, i}=\operatorname{sign}\left(I_{1, i}\right), \\
& D_{i} \leq 0, \quad a_{2, i}=-1, \quad \text { and } a_{1, i}=\operatorname{sign}\left(I_{2, i}\right) .
\end{aligned}
$$

The absolute value of the correlator's output is used here because it is easy to implement and it is found to give better performance than detection with two thresholds. The performance of the proposed CIM-SS system is investigated in the following section.

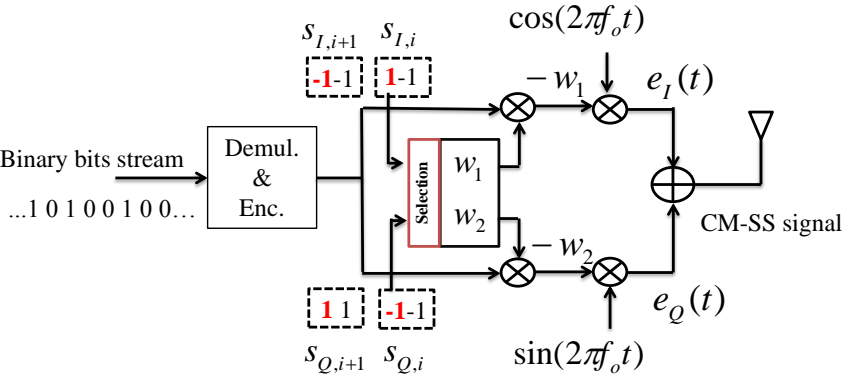

(a) Transmitter structure for CIM system

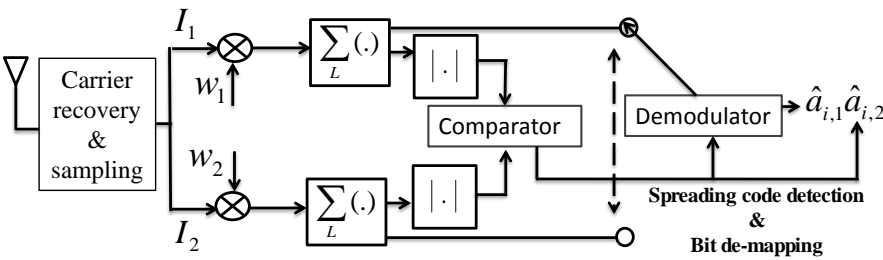

(b) In phase receiver structure for CIM system

Fig. 1. System model

\section{CIM PERFORMANCE ANALYSIS}

In this section, we first derive the BER of the proposed CIM-SS system over AWGN channel. Then, we analyse the throughput, energy consumption, and spreading complexity compared to the conventional SS-QPSK system.

1) BER: The total BER $P_{T}$ of this system is function of the BER of the transmitted bit $P_{e b}$ and the BER the mapped bit $P_{e c}$, which corresponds to the error probability of spreading code detection. Since there is one transmitted bit $a_{1, i}$ and one mapped bit $a_{2, i}$ with each spreading code, the total BER $P_{T}$ of the CIM-SS system is given by

$$
P_{T}=\frac{1}{2} P_{e b}+\frac{1}{2} P_{e c} .
$$

From (5), we see that the error of the transmitted bit $a_{1, i}$ depends on the comparator decision and occurs in two different cases. The first case is when there is no error in the spreading code detection, but the transmitted bit $a_{1, i}$ is detected with an error. The second case is when there is an error in the code detection since $a_{1, i}$ is detected based on the sign of the wrong correlator output. Therefore, the BER $P_{e b}$ of the transmitted bit $a_{1, i}$ is given by

$$
P_{e b}=P_{s s}\left(1-P_{e c}\right)+\frac{1}{2} P_{e c},
$$

where $P_{s s}=0.5 \operatorname{erfc}\left(\sqrt{E_{b} / N_{0}}\right)$ is the BER for the conventional DS-SS (i.e DS-SS with QPSK) over AWGN and erfe is the complementary error function [11]. The two terms in (7) represents the two error cases respectively. Also, the factor 0.5 represents the fact that with error in the code detection, the bit value detected based on the sign of the wrong correlator output still matches the transmitted bit $a_{1, i}$ half of the time.

Computing (6) and (7) requires finding the error probability of spreading code detection $P_{e c}$. As seen in (5), the decision variable $D_{i}$ of the in-phase signal is compared to zero and the result determines the spreading code. The decision variable $D_{i}$ can be approximated as a nearly Gaussian random variable [12] and thus the error probability is expressed in terms of 
the Q-function. We first compute the mean and variance of the decision variable $D_{i}$. Since the outputs in (3) and (4) are symmetric, we can consider one case and the other one is similar. Let us assume the code $w_{1}$ is transmitted, i.e. $a_{2, i}=1$, then $D_{i}$ is given by

$$
D_{i}=\left|I_{1, i}\right|-\left|I_{2, i}\right|=\left|E_{b}+n_{1}\right|-\left|n_{2}\right|,
$$

then the mean $E\left\{D_{i}\right\}$ of $D_{i}$ is given as

$$
\mathrm{E}\left\{D_{i}\right\}=\mathrm{E}\left\{\left|E_{b}+n_{1}\right|\right\}-\mathrm{E}\left\{\left|n_{2}\right|\right\} .
$$

The two terms in (9) are independent because the noise signal is multiplied by two different Walsh codes. We can write the first term as $\mathrm{E}\left\{\left|E_{b}+n_{1}\right|\right\}=\mathrm{E}\{|X|\}$ where $X=E_{b}+n_{1}$ is a random variable distributed following normal distribution with mean $E_{b}$ and variance $E_{b} N_{0} / 2$, i.e. $X \sim \mathcal{N}\left(E_{b}, E_{b} N_{0} / 2\right)$, then mean $\mathrm{E}\{|X|\}$ can be developed as

$$
\begin{aligned}
\mathrm{E}\{|X|\} & =\frac{1}{\sqrt{\pi E_{b} N_{0}}} \int_{-\infty}^{+\infty}|X| e^{-\frac{\left(X-E_{b}\right)^{2}}{E_{b} N_{0}}} d X \\
& =\sqrt{\frac{E_{b} N_{0}}{\pi}} e^{\left(-\frac{E_{b}}{N_{0}}\right)}+E_{b} \operatorname{erf}\left(\sqrt{\frac{E_{b}}{N_{0}}}\right) .
\end{aligned}
$$

Moreover, $\left|n_{2}\right|$ follows half-normal distribution with mean $\mathrm{E}\left\{\left|n_{2}\right|\right\}=\sqrt{\frac{E_{b} N_{0}}{\pi}}$ [13]. Hence, the mean of the decision variable $D_{i}$ is given by

$\mathrm{E}\left\{D_{i}\right\}=\left(e^{\left(-\frac{E_{b}}{N_{0}}\right)}-1\right) \sqrt{\frac{E_{b} N_{0}}{\pi}}+E_{b} \operatorname{erf}\left(\sqrt{\frac{E_{b}}{N_{0}}}\right)$.

The variance $V\left\{D_{i}\right\}$ of the decision variable is expressed as $V\left\{D_{i}\right\}=\mathrm{E}\left\{D_{i}^{2}\right\}-\mathrm{E}\left\{D_{i}\right\}^{2}=\mathrm{E}\left\{E_{b}^{2}\right\}+\mathrm{E}\left\{n_{1}^{2}\right\}+\mathrm{E}\left\{n_{2}^{2}\right\}$

$$
-2 \mathrm{E}\left\{\left|E_{b}+n_{1}\right|\right\} \mathrm{E}\left\{\left|n_{2}\right|\right\}-\mathrm{E}\left\{D_{i}\right\}^{2} \text {. }
$$

Equation (12) is obtained because the in-phase signal is considered as the the independent noise components. Moreover, this assumption allows us to express the squared absolute values in this expression as $E\left\{|x|^{2}\right\}=E\left\{x^{2}\right\}$. By substituting (10) and (11) into (12) and after some manipulations, the total variance can be expressed as

$$
\begin{aligned}
V\left\{D_{i}\right\} & =E_{b}^{2}-\sqrt{\frac{E_{b} N_{0}}{\pi}}\left(e^{\left(-\frac{E_{b}}{N_{0}}\right)}\left(1+2 \sqrt{\frac{E_{b} N_{0}}{\pi}}\right)-1\right) \\
& +E_{b}\left(N_{0}+\operatorname{erf}\left(\sqrt{\frac{E_{b}}{N_{0}}}\right)\left(1-2 \sqrt{\frac{E_{b} N_{0}}{\pi}}\right)\right) \cdot(13)
\end{aligned}
$$

Thus, the error probability of spreading code detection under Gaussian approximation can be expressed as [11]

$$
P_{e c} \approx \frac{1}{2} \operatorname{erfc}\left(\frac{E\left[D_{i}\right]}{\sqrt{2 V\left[D_{i}\right]}}\right)
$$

The closed-from expression of the error probability $P_{e c}$ is obtained by substituting (11) and (13) in (14). Finally, the total BER of the CIM-SS system over AWGN channel is obtained by substituting (14) and (7) in (6).
2) Throughput: Typically, the throughput is defined as the number of correct bits that a user receives per unit time and thus can be written as [11]

$$
R_{t}=\log _{2} M \frac{\left(1-\mathrm{P}_{\mathrm{T}}\right)}{T},
$$

where, $M$ is the modulation order, $T$ is the transmission time, $P_{T}$ is the error probability, and $\left(1-\mathrm{P}_{\mathrm{T}}\right)$ is the correct bits received during time $T$. Both SS-QPSK and CIM-SS systems have the same bit duration $T=L T_{c}$. However, two bits are transmitted over $T$ with SS-QPSK system while four bits are transmitted for the CIM.

3) Energy efficiency, data rate, and spreading complexity: In the proposed CIM-SS scheme, only half of the information bits are directly transmitted. With each transmitted bit in the in-phase and quadrature-phase components, another bit is conveyed in the spreading code selection. This process saves $50 \%$ of the total used energy and doubles the data rate of the system as compared to the conventional SS-QPSK. The proposed scheme is a good choice from the energy perspective when BPSK is not sufficient, as it outperforms other schemes with the same bit rate.

The spreading complexity of the schemes is evaluated here by the number of spreading/de-spreading operations required to transmit $N_{b}$ bits. At the transmitter side, the CIM-SS scheme requires one spreading operation to transmit one bit which is the same number of operations required for SSQPSK case. At the receiver side, two de-spreading operations are required for the CIM-SS instead of one operation as in the SS-QPSK system. However, to transmit $N_{b}$ bits, the CIM-SS system requires $N_{b} / 2$ spreading operations at the transmitter and $N_{b}$ de-spreading operations at the receiver with total of $3 / 2 N_{b}$ operations, while SS-QPSK system requires $N_{b}$ spreading operations and $N_{b}$ de-spreading operations. Therefore, if we neglect the comparator complexity, the CIMSS systems is approximately $25 \%$ less complex than the SSQPSK scheme. This lower spreading complexity also reduces the energy consumption due to computations.

\section{Simulation RESUlts}

Fig. 2 shows the theoretical BER expression given in (6) for the CIM-SS over AWGN channel and compares the obtained performance with the conventional SS-QPSK system. Since the CIM and SIM are conceptually similar with the difference in the new dimension used to code the additional bits, we compared the BER performance of the proposed CIM-SS system to the corresponding performance of the ESIM-OFDM [8] with both M-QAM and M-PSK schemes. In addition, since the CIM-SS system transmits 4 bits per symbol, we compared it to an SS-M-PSK and ESIM-OFDM systems having the same number of bits per symbol which is $M=16$ in this case. To give an idea about the expected performance under different channels, this figure also shows the simulated performance of the two scheme under flat fading Rayleigh channel with average power gain equal to 0.5 . As we can see from the figure, there is a small difference between the theoretical error expression $P_{T}$ and simulation result due to the approximation of the decision variable $D_{i}$ as a Gaussian random variable. However, 


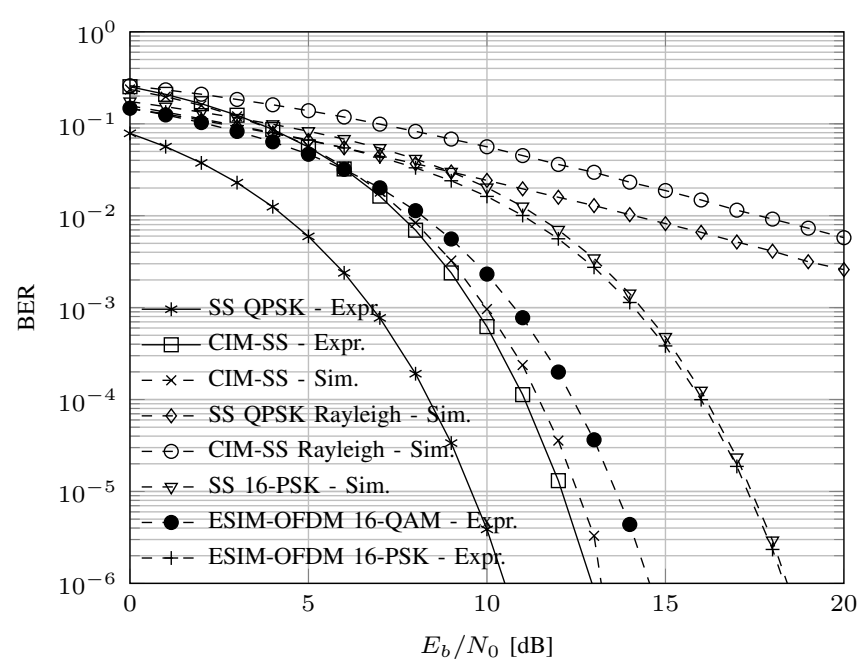

Fig. 2. BER for SS-QPSK, SS-16-PSK, CIM-SS and ESIM-OFDM schemes over an AWGN and Rayleigh fading channels for spreading gain equal to 16 .

the given results validate our Gaussian approximation of the decision variable as an acceptable assumption which simplify the theoretical analysis with just a small gap in the final result. In addition, we can see that the conventional SS-QPSK system outperforms our system with about $3 \mathrm{~dB}$ for both AWGN and Rayleigh channels. Based on our analytical analysis given in (6), this $3 \mathrm{~dB}$ degradation comes from the error in the code detection. However, the performance degradation gap between the SS-QPSK and higher order modulation with four bits per symbol like SS-16-PSK over AWGN channel is $8 \mathrm{~dB}$ [11] which is higher than the $3 \mathrm{~dB}$ gap with our proposed system. This is because that the mapped bits does not reduce the Euclidean distance between the transmitted bits as in the conventional modulation. Therefore, our proposed CIMSS system increase the data rate with $5 \mathrm{~dB}$ gain in term of BER than the equivalent conventional higher order modulation schemes. Finally, we can also see that our CIM-SS system outperforms the ESIM-OFDM for the same number of bits per symbol.

Fig. 3 compares the throughput for the proposed CIMSS and the conventional SS-QPSK systems obtained by (15). As shown in the figure, the CIM-SS scheme offers a higher throughput than the SS-QPSK because, for the same period $T$, four bits are sent over the channel in the CIM-SS where two bits are physically transmitted and two bits are mapped with the spreading code. Consequently, the proposed system outperform the SS-QPSK system and save half of the transmitted energy while being $25 \%$ less complex.

Finally, since any enhancement in CIM-SS system performance passes through improving the code detection algorithm, future work will focus on this area to improve the total BER performance.

\section{CONCLUSION}

A new high data rate and energy efficient spread spectrum communication system was presented in this paper. It is based on an original idea to increase the data rate by using new dimension (the spreading code) to map the information bits. The analysis showed that this system can save $50 \%$ of the used bit energy and increase the throughput while being less complex as compared to the conventional SS-QPSK system.

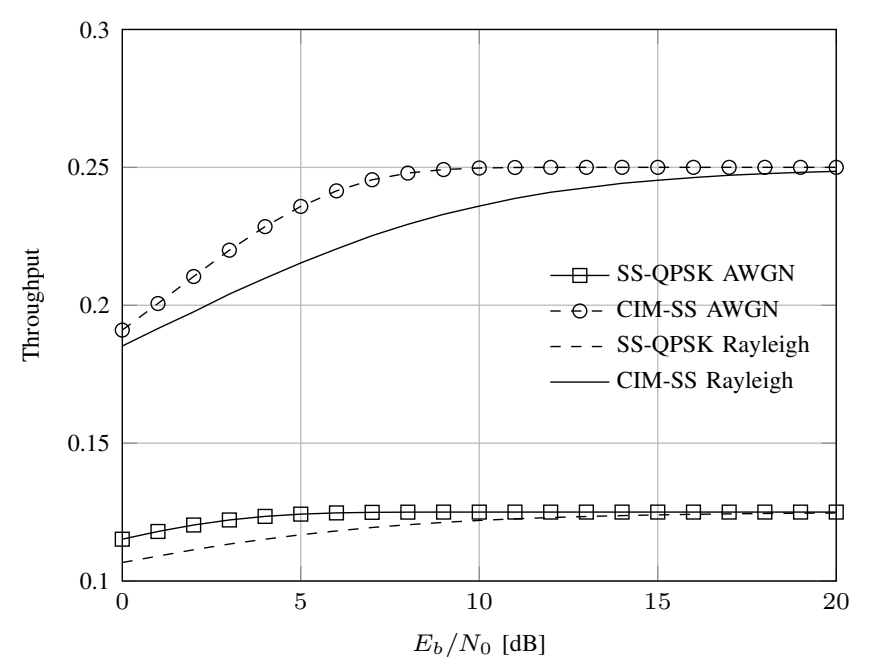

Fig. 3. Throughput of the CIM-SS and SS-QPSK schemes over an AWGN and Rayleigh fading channels for spreading gain equal to 16 .

On the other hand, we have shown that the SS-QPSK system outperforms our system with $3 \mathrm{~dB}$ in term of BER. However, this gap is lower than the performance degradation gap of 8 $\mathrm{dB}$ between SS-QPSK and modulation scheme with four bits per symbol like SS-16-PSK over AWGN channel.

\section{REFERENCES}

[1] R. Baldemair, E. Dahlman, G. Fodor, G. Mildh, S. Parkvall, Y. Selen, H. Tullberg, and K. Balachandran, "Evolving Wireless Communications: Addressing the Challenges and Expectations of the Future," IEEE Vehicular Technology Magazine, vol. 8, no. 1, pp. 24-30, Mar. 2013.

[2] R. Cavallari, F. Martelli, R. Rosini, C. Buratti, and R. Verdone, "A Survey on Wireless Body Area Networks: Technologies and Design Challenges," IEEE Communications Surveys Tutorials, vol. PP, no. 99, pp. 1-23, 2014.

[3] I. Akyildiz, W. Su, Y. Sankarasubramaniam, and E. Cayirci, "A Survey on Sensor Networks," IEEE Communications Magazin, vol. 40, no. 8, pp. 102-114, Aug. 2002.

[4] M. Xiao and T. M. Aulin, "Serially concatenated continuous phase modulation with convolutional codes over rings," IEEE Trans. on Communications, vol. 54, no. 8, pp. 1387-1396, Aug. 2006.

[5] D. Gesbert, M. Shafi, D. shan Shiu, P. Smith, and A. Naguib, "From Theory to Practice: An Overview of MIMO Space-Time Coded Wireless Systems," IEEE Journal on Selected Areas in Communications, vol. 21, no. 3, pp. 281-302, Apr. 2003.

[6] M. Di Renzo, H. Haas, A. Ghrayeb, S. Sugiura, and L. Hanzo, "Spatial Modulation for Generalized MIMO: Challenges, Opportunities, and Implementation," Proceedings of the IEEE, vol. 102, no. 1, pp. 56-103, Jan. 2014.

[7] R. Abu-alhiga and H. Haas, "Subcarrier-Index Modulation OFDM," in Personal, Indoor and Mobile Radio Communications, 2009 IEEE 20th International Symposium on, Sep. 2009, pp. 177-181.

[8] D. Tsonev, S. Sinanovic, and H. Haas, "Enhanced subcarrier index modulation (SIM) OFDM," in IEEE GLOBECOM Workshops, Dec. 2011, pp. 728-732.

[9] T. Zhao, Z. Yang, and Y. Zhao, "Partial-code-aided scheme for narrowband interference suppression in direct-sequence spread spectrum systems," IET Communications, vol. 4, no. 18, pp. 2240-2251, Dec. 2010.

[10] X. Wu, Z. Yang, J. Yan, and J. Cui, "Low-rate turbo-hadamard coding approach for narrow-band interference suppression," in IEEE International Conference Communications (ICC), Sydney, Australia, Jun. 2014, pp. 2130-2134.

[11] D. Tse and P. Viswanath, Fundamentals of Wireless Communication. Cambridge university press, 2005.

[12] D. Morales-Jimenez, R. H. Y. Louie, M. R. McKay, and Y. Chen, "Multiple-Antenna Signal Detection in Cognitive Radio Networks with Multiple Primary User Signals," submitted arXiv:1405.6408v1, May 2014.

[13] A. Papoulis, Probability, Random Variables, and Stochastic Processes. McGraw-Hill, 1991. 